\title{
THE DUAL VARIABLE METHOD FOR THE SOLUTION OF COMPRESSIBLE FLUID FLOW PROBLEMS*
}

\author{
J. BURKARDT $\dagger$, C. HALL $\dagger$ AND T. PORSCHING $\dagger$
}

\begin{abstract}
Discretizations of the Navier-Stokes equations describing a compressible flow problem can be viewed as systems defining flows on an associated network. This observation provides a means of economizing on their numerical solution.
\end{abstract}

Key words. Navier-Stokes, networks, dual variable, compressible fluid

AMS(MOS) subject classifications. 05C38, 65M10, 76D05

1. Introduction. The dual variable method [1] is a means of economizing on the cost of solving the linear or nonlinear systems that arise in certain discretizations of the Navier-Stokes equations. A matrix transformation is introduced which significantly reduces the size of the system which must be solved. For the finite difference discretization of the two-dimensional, incompressible Navier-Stokes equations studied in [1], [2] this reduction amounts to a factor of 3. A key element of the implementation of this transformation is the construction of a cycle vector basis for an associated network.

In this paper we extend the dual variable method to compressible flow problems. This again involves the use of network theory.

The system of partial differential equations in two spatial dimensions $(x, y)$ and time $t$ describing the compressible (barotropic) flow problem of concern is:

$$
\begin{aligned}
& \frac{\partial \rho}{\partial t}+\nabla \cdot(\rho \mathbf{q})=0, \\
& \rho \frac{\partial q}{\partial t}+\rho(\mathbf{q} \cdot \nabla) \mathbf{q}+\nabla p-\mu\left[\nabla^{2} \mathbf{q}+\frac{1}{3} \nabla(\nabla \cdot \mathbf{q})\right]=\mathbf{F}
\end{aligned}
$$

where $\mathbf{q}=(u, v)$ is the velocity vector, $p$ is pressure, $\mu$ is viscosity, $\mathbf{F}$ is a vector that includes elevation and wall friction effects, and the density $\rho$ is determined by a state equation

$$
\rho=\rho(p) .
$$

Equation (1) is referred to as the continuity equation and equation (2) as the momentum equation.

We assume that appropriate boundary conditions and an initial condition are specified so that (1)-(3) have a unique solution in a flow region $\Omega$. Typical boundary conditions are the specification of the pressure or velocity on each segment of the boundary $\Omega$.

In $\S 2$, we present details of a discretization of (1)-(3). The matrix transformations involved in the dual variable method are given in $\S 3$, and a network or physical interpretation of the dual variable transformation is given in $\S 4$. Section 5 contains numerical results.

* Received by the editors April 8, 1985, and in revised form October 7, 1985. This work was supported by the Air Force Office of Scientific Research under grant 84-0131.

$\dagger$ Institute for Computational Mathematics and Applications, Department of Mathematics and Statistics, University of Pittsburgh, Pittsburgh, Pennsylvania 15260. 
2. The finite difference equations. There are several consistent finite difference discretizations that are available to approximate (1)-(2). We choose the following scheme based on the MAC placement of variables [3] in which a pressure is associated with the center of a control volume or mesh box and the component of velocity normal to a control volume side is associated with the center of that side (Fig. 1). Let $U, V$, $P$ be the finite difference approximations to the mass velocities $\rho u, \rho v$, and pressure $p$ respectively. A superscript $m$ designates the $m$ th time level.
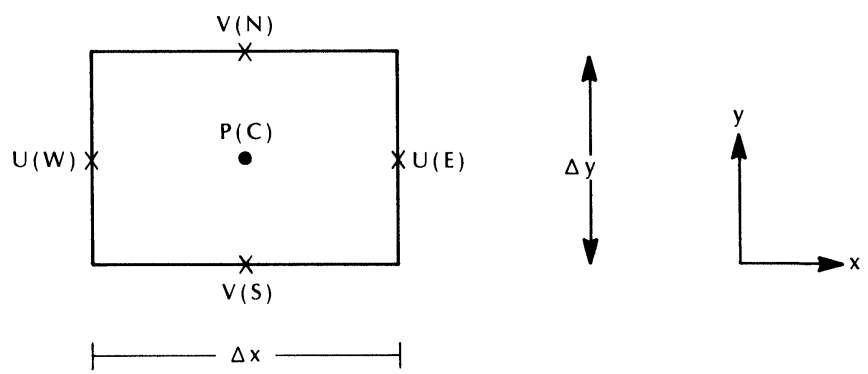

FIG. 1. A control volume with MAC placement of variables and compass designations.

In the continuity equation, we expand the time derivative via the chain rule, and use backward differencing and centered differencing on the temporal and divergence terms respectively. The discrete equation is then of the form:

$$
\frac{U_{E}^{m+1}-U_{W}^{m+1}}{\Delta x}+\frac{V_{N}^{m+1}-V_{S}^{m+1}}{\Delta y}+\left(\frac{\partial \rho}{\partial p}\right)_{C}^{m}\left(\frac{P_{C}^{m+1}-P_{C}^{m}}{\Delta t}\right)=0 .
$$

The momentum equations are discretized as in [1], [2] using upwind differences for the convective terms $(\mathbf{q} \cdot \nabla) \mathbf{q}$, centered differences for the pressure gradient $\nabla p$ and the viscous terms $\left[\nabla^{2} \mathbf{q}+\frac{1}{3} \nabla(\nabla \cdot \mathbf{q})\right]$, and backward differences for the temporal derivative $\partial \mathbf{q} / \partial t$. The finite difference system resulting from (4) and the discrete momentum equations can be written as $(N+L)$ equations

$$
\begin{aligned}
& A \mathbf{V}^{m+1}+\mathbf{W}_{P}^{m+1}=0, \\
& Q_{m} \mathbf{V}^{m+1}-\Delta t A^{T} \mathbf{P}^{m+1}=\mathbf{b}_{1}^{m} .
\end{aligned}
$$

Here the $N \times 1$ vector $\mathbf{P}^{m+1}$, and $L \times 1$ vector $\mathbf{W}^{m+1}$ contain the unknown pressures and velocities respectively, $\mathbf{V}^{m+1} \equiv D_{1} \mathbf{W}^{m+1}, D_{1}$ a diagonal matrix with $\left[D_{1}\right]_{i i}=\Delta x$ if $i$ corresponds to $N$ or $S$ and $\left[D_{1}\right]_{i i}=\Delta y$ if $i$ corresponds to $E$ or $W$ (note that $\Delta x$ $(\Delta y)$ may vary from one column (row) of mesh boxes to the next),

$$
\mathbf{W}_{P}^{m+1} \equiv \Delta t Q_{22}^{-1} \mathbf{P}^{m+1}-\mathbf{S}^{m}
$$

where $Q_{22}^{-1}$ is the diagonal matrix

$$
Q_{22}^{-1}=\operatorname{diag}\left[\frac{\Delta x \Delta y}{\Delta t^{2}}\left(\frac{\partial \rho}{\partial p}\right)_{C}^{m}\right],
$$

$$
\left[\mathbf{S}^{m}\right]_{C}=\frac{\Delta x \Delta y}{\Delta t}\left[\left(\frac{\partial \rho}{\partial p}\right)_{C}^{m} P_{C}^{m}\right]+\text { boundary mass velocities. }
$$

The $N \times L$ matrix $A$ contains 0 's, 1 's and -1 's and can be interpreted as an incidence matrix of an associated network as described in [1], [4], [5]. The $L \times L$ matrix $Q_{m}$ contains the finite difference coefficients of the discrete convective and 
viscous terms in the momentum equations as well as the temporal term. The $L \times 1$ vector $\mathbf{b}_{1}^{m}$ contains boundary data as well as contributions of the body force and friction terms.

If we let $\mathbf{Z}^{m+1}=\left[\mathbf{V}^{m+1}, \mathbf{W}_{P}^{m+1}\right]$ and

$$
B=\left(A \mid I_{N}\right),
$$

where $I_{N}$ denotes the identity of order $N$, then the discrete continuity equation is of the generic form

$$
B Z^{m+1}=0 .
$$

We may think of the vector $\mathbf{W}_{P}^{m+1}$ as "pseudo mass flows." Equations (6) and (7) then combine to give

$$
\left[\begin{array}{cc}
Q_{m} & 0 \\
0 & Q_{22}
\end{array}\right]\left[\begin{array}{c}
\mathbf{V}^{m+1} \\
\mathbf{W}_{P}^{m+1}
\end{array}\right]=\Delta t\left[\begin{array}{c}
A^{T} \\
I_{N}
\end{array}\right] \mathbf{P}^{m+1}+\left[\begin{array}{c}
\mathbf{b}_{1}^{m} \\
-Q_{22} \mathbf{S}^{m}
\end{array}\right]
$$

or

$$
\left[\begin{array}{cc}
Q_{m} & 0 \\
0 & Q_{22}
\end{array}\right] \mathbf{Z}^{m+1}=\Delta t B^{T} \mathbf{P}^{m+1}+\mathbf{k}^{m}
$$

Thus, it is required to solve the $2 N+L$ equations (11)-(12) for $\mathbf{Z}^{m+1}$ and $\mathbf{P}^{m+1}$. In the next section we show how to obtain an equivalent system from which the pressure vector $\mathbf{P}^{m+1}$ has been eliminated.

3. The dual variable transformation. The dual variable method has been used successfully in the treatment of certain finite difference and finite element discretizations of the equations of incompressible flow [1], [2]. With regard to the current system (11), (12) of compressible flow equations, the method consists of the following steps.

Step 1. Find a basis $\left[\mathbf{C}_{1}, \mathbf{C}_{2}, \cdots, \mathbf{C}_{d}\right]$ for the null space of $B$ and form the $(L+N) \times d$ matrix $C$ with $C_{i}$ as its $i$ th column. Then

$$
B C=0
$$

and

$$
\mathbf{Z}^{m+1}=C \mathbf{X}^{m+1}
$$

for some $d \times 1$ vector $\mathbf{X}^{m+1}$.

Step 2. Substitute $\mathbf{Z}^{m+1}$ as defined by (14) into (12) to obtain

$$
\left[\begin{array}{cc}
Q_{m} & 0 \\
0 & Q_{22}
\end{array}\right] C \mathbf{X}^{m+1}=\Delta t B^{T} \mathbf{P}^{m+1}+\mathbf{k}^{m} .
$$

Step 3. Multiply (15) by $C^{T}$ and use the orthogonality of $B^{T}$ and $C^{T}$ to obtain the $d \times d$ system

$$
C^{T}\left[\begin{array}{cc}
Q_{m} & 0 \\
0 & Q_{22}
\end{array}\right] C \mathbf{X}^{m+1}=C^{T} \mathbf{k}^{m} .
$$

The matrix transformation in (16) is called the dual variable transformation and (16) is called the dual variable system.

Step 4. Solve (16) for $\mathbf{X}^{m+1}$ and recover the velocities $\mathbf{V}^{m+1}$ and pseudo velocities $\mathbf{W}_{P}^{m+1}$ from (14). 
Step 5. Recover the pressures from the pseudo velocities using (7), noting that $Q_{22}$ is diagonal.

The inherent advantage of the dual variable method is the reduction in the size $(L+N$ to $d)$ of the system to be solved at each time step. Efficient algorithms for computing well conditioned sparse bases for null spaces have been studied by Berry, Heath, Kaneko, Lawo, Plemmons and Ward [6]. This latter approach involves a matrix factorization. As we now show, $C$ can be constructed without the need to solve any system of equations.

It is clear that the $N \times(N+L)$ matrix in (10) is of rank $N$. We have then the dimension, $d$, of the null space of $B$ is $L$, the number of columns minus the rank. Moreover, a basis for this null space is immediately provided by the columns of the matrix $C$ defined as follows,

$$
C \equiv\left[\begin{array}{c}
I_{L} \\
-A
\end{array}\right]
$$

Substituting (17) into (16), we obtain the $L \times L$ system

$$
\left(Q_{m}+A^{T} Q_{22} A\right) \mathbf{X}^{m+1}=\mathbf{b}_{1}^{m}+A^{T} Q_{22} \mathbf{S}^{m}
$$

as the dual variable system. But by (14) and (17), $\mathbf{V}^{m+1}=\mathbf{X}^{m+1}$, and

$$
\mathbf{W}_{P}^{m+1}=-A \mathbf{X}^{m+1} \text {. }
$$

Hence, the unknown velocities actually satisfy (18) and the pseudo flows that are needed to recover the pressures via (7) are given by (19).

4. A network interpretation. As in the case of the discrete divergence matrix $\boldsymbol{A}$, the augmented matrix $B$ can also be interpreted as the incidence matrix of a directed network $T$. The geometric realization $G(T)$ is constructed as follows:

The nodes of $G(T)$ are the mesh box (control volume) centers and the interior links connect nodes of contiguous mesh boxes. The boundary links of $G(T)$ are links normal to segments of the boundary of the flow region where a pressure is specified. All links are oriented in the positive sense of the $x$ or $y$ axis, respectively. So far this planar network is precisely the network used in the dual variable formulation of incompressible flow problems [1], [4], [5], and $A$ is its incidence matrix. However, we now add links which emanate from each mesh box center (node) and terminate at a fictitious node. These links are all directed toward this fictitious node. The $N$ unknowns $\left[\mathbf{W}_{P}^{m+1}\right]_{i}, i=1,2, \cdots, N$ in (7) are then thought of as pseudo-flows on these latter links just as the $L$ unknowns $\left[\mathbf{V}^{m+1}\right]_{j}, j=1, \cdots, L$ are flows on the links connecting mesh box centers. The $N \times(N+L)$ matrix $B$ is the node-link incidence matrix for the network so constructed. That is,

$$
[B]_{k l}=\left\{\begin{aligned}
+1 & \text { if link } l \text { is directed away from node } k \\
-1 & \text { if link } l \text { is directed toward node } k \\
0 & \text { otherwise. }
\end{aligned}\right.
$$

Equation (11) then states that at each node the total "flow" is in balance. In Fig. 2 there are $N=14$ unknown pressures (also 14 unknown pseudo flows) and $L=22$ unknown velocities. At each of the $N=14$ nodes the sum of the flows and pseudo flows is forced to be zero. 


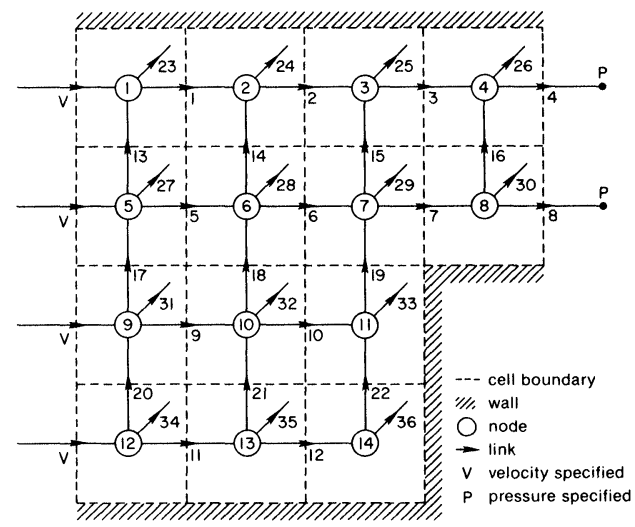

FiG. 2. Flow region decomposed into 14 flow cells showing associated network of $N=14$ nodes and $N+L=36$ links.

The $14 \times 22$ matrix $A$ is given by

\begin{tabular}{|c|c|c|c|c|c|c|c|c|c|c|c|c|c|c|c|c|c|c|c|c|c|c|}
\hline 1 & 2 & 3 & 4 & 5 & 6 & 7 & 8 & 9 & 10 & 11 & 12 & 13 & 14 & 15 & 16 & 17 & 18 & 19 & 20 & 21 & 22 & \\
\hline 1 & 0 & 0 & 0 & 0 & 0 & 0 & 0 & 0 & 0 & 0 & 0 & -1 & 0 & 0 & 0 & 0 & 0 & 0 & 0 & 0 & 0 & 1 \\
\hline-1 & 1 & 0 & 0 & 0 & 0 & 0 & 0 & 0 & 0 & 0 & 0 & 0 & -1 & 0 & 0 & 0 & 0 & 0 & 0 & 0 & 0 & 2 \\
\hline 0 & -1 & 1 & 0 & 0 & 0 & 0 & 0 & 0 & 0 & 0 & 0 & 0 & 0 & -1 & 0 & 0 & 0 & 0 & 0 & 0 & 0 & 3 \\
\hline 0 & 0 & -1 & 1 & 0 & 0 & 0 & 0 & 0 & 0 & 0 & 0 & 0 & 0 & 0 & -1 & 0 & 0 & 0 & 0 & 0 & 0 & 4 \\
\hline 0 & 0 & 0 & 0 & 1 & 0 & 0 & 0 & 0 & 0 & 0 & 0 & 1 & 0 & 0 & 0 & -1 & 0 & 0 & 0 & 0 & 0 & 5 \\
\hline 0 & 0 & 0 & 0 & -1 & 1 & 0 & 0 & 0 & 0 & 0 & 0 & 0 & 1 & 0 & 0 & 0 & -1 & 0 & 0 & 0 & 0 & 6 \\
\hline 0 & 0 & 0 & 0 & 0 & -1 & 1 & 0 & 0 & 0 & 0 & 0 & 0 & 0 & 1 & 0 & 0 & 0 & -1 & 0 & 0 & 0 & 7 \\
\hline 0 & 0 & 0 & 0 & 0 & 0 & -1 & 1 & 0 & 0 & 0 & 0 & 0 & 0 & 0 & 1 & 0 & 0 & 0 & 0 & 0 & 0 & 8 \\
\hline 0 & 0 & 0 & 0 & 0 & 0 & 0 & 0 & 1 & 0 & 0 & 0 & 0 & 0 & 0 & 0 & 1 & 0 & 0 & -1 & 0 & 0 & 9 \\
\hline 0 & 0 & 0 & 0 & 0 & 0 & 0 & 0 & -1 & 1 & 0 & 0 & 0 & 0 & 0 & 0 & 0 & 1 & 0 & 0 & -1 & 0 & 10 \\
\hline 0 & 0 & 0 & 0 & 0 & 0 & 0 & 0 & 0 & -1 & 0 & 0 & 0 & 0 & 0 & 0 & 0 & 0 & 1 & 0 & 0 & -1 & 11 \\
\hline 0 & 0 & 0 & 0 & 0 & 0 & 0 & 0 & 0 & 0 & 1 & 0 & 0 & 0 & 0 & 0 & 0 & 0 & 0 & 1 & 0 & 0 & 12 \\
\hline 0 & 0 & 0 & 0 & 0 & 0 & 0 & 0 & 0 & 0 & -1 & 1 & 0 & 0 & 0 & 0 & 0 & 0 & 0 & 0 & 1 & 0 & 13 \\
\hline 0 & 0 & 0 & 0 & 0 & 0 & 0 & 0 & 0 & 0 & 0 & -1 & 0 & 0 & 0 & 0 & 0 & 0 & 0 & 0 & 0 & & \\
\hline
\end{tabular}

and the matrix $B=\left(A \mid I_{14}\right)$ is the $14 \times 36$ incidence matrix for the network shown in Fig. 2.

As defined by (17) the matrix $C$ may be interpreted as a fundamental cycle basis (cf. [7]). Each column of $C$ is a cycle vector for the network $T$. For example, for the network of Fig. 2, the sixth column is

$$
\left[\operatorname{Col}_{6}(C)\right]_{i}=\left\{\begin{aligned}
1, & i=6 \quad(\text { link } 6) \\
-1, & i=28 \quad(L+\text { start node for link 6), } \\
1, & i=29 \quad(L+\text { end node for link 6), } \\
0 & \text { otherwise }
\end{aligned}\right.
$$

In general, if link $j$ is not a boundary link and is incident from node $l$ to node $k$, then

$$
\left[\operatorname{Col}_{j}(C)\right]_{i}=\left\{\begin{aligned}
1, & i=j, k+L, \\
-1, & i=l+L, \\
0 & \text { otherwise. }
\end{aligned}\right.
$$

If link $j$ is a boundary link, then this definition yields only two nonzero entries in the $j$ th column of $C$ since one of the nodes $l$ or $k$ does not exist. 
Finally, we can conveniently use the network concept to determine the sparsity of the dual variable system (16). We observe that

$$
\left(A^{T} Q_{22} A\right)_{j r}=\left(Q_{22}\right)_{l l} A_{l r}-\left(Q_{22}\right)_{k k} A_{k r}
$$

where $j, k$ and $l$ are related as in Fig. 3. It follows that the right side of (21) is nonzero only when $r$ corresponds to cycles containing those interior and boundary links that are incident to or from nodes $k$ and $l$. Consequently, the $j$ th equation of (18) is coupled only to itself and the eight other equations corresponding to the cycles containing the links shown in Fig. 4. That is, dual variable $j$ associated with cycle $j$ is coupled to at most 8 other dual variables associated with the cycles (or equivalently links) illustrated. Hence the coefficient matrix in (19) has at most 9 nonzero entries in each row, and, with suitable ordering of the links, may be solved as a banded matrix.

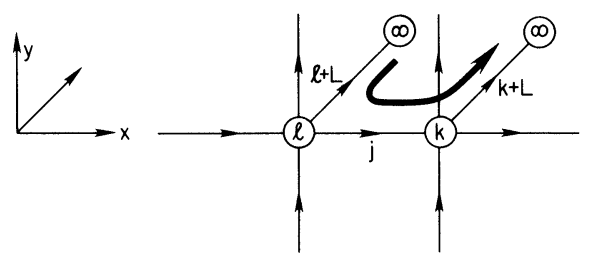

FIG. 3. The jth cycle associated with link $j$. The fictitious node is labeled $\infty$.

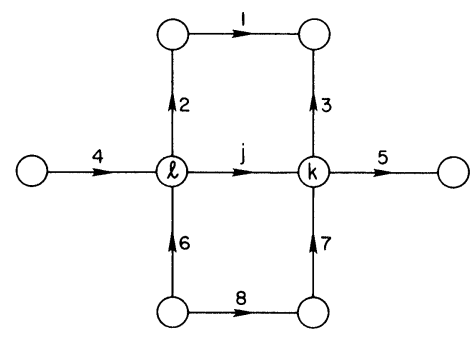

FIG. 4. Stencil for the dual variable system (19). Each link corresponds to a cycle and a dual variable.

The potential computational advantage of the dual variable method lies in the reduction of the size of the discrete Navier-Stokes system from $N+L$ to $L$ equations and unknowns. Although the sparsity of the dual variable system (18) is less than that of the primitive system (5)-(6), the decrease is slight. The maximum number of nonzero couplings per equation increases from 7 in system (5)-(6) to 9 in system (18). Since any implementation of the method depends strongly on such imponderables as solution algorithm, data structure, computer architecture, etc., it is not possible to state unequivocally that the dual variable method will always produce dramatic reductions in running times. However, it is quite natural to expect that, "all other things being equal," the slight increase in the complexity of the dual variable system is more than offset by its decrease in size. Moreover, apart from any computational advantage the method may have, it provides additional insight into the physics of compressible flow through a novel interpretation of that phenomenon in terms of network concepts.

5. Example: aircraft cavity. Aircraft that are used for observation sometimes have cavities or compartments that open directly to the atmosphere. Figure 5 illustrates a two-dimensional model of such a cavity with dimensions as indicated. The blockage in the cavity simulates instrumentation used during the observations. The spoiler ahead 


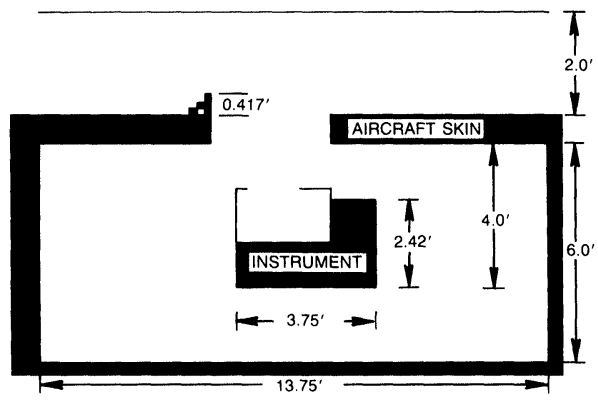

FIG. 5. Flow region for aircraft cavity.

of the cavity opening is used to divert the air flow so as to stabilize the flow in front of the instrumentation. We indicate a solid spoiler, although a porous spoiler may also be used. We assume the aircraft is flying at mach 0.75 and an altitude of 37,000 feet. The ambient pressure of 2.7 psi is specified at the downstream flow region boundary, and upstream the inlet velocity profile is given by:

$$
u=750 \begin{cases}(y / 0.417)^{1 / 7}, & 0 \leqq y \leqq 0.417, \\ 1, & y \geqq 0.417,\end{cases}
$$

where $y$ is the distance from the aircraft skin. All walls are assumed to be no-slip walls.

The flow region was subdivided into $N=1167$ flow cells and there are $N=1167$ unknown pressures. The number of unknown velocities is $L=2206$. The primitive system (5)-(6) is of dimension $L+N=3373$ while the dual variable system (19) is of dimension $L=2206$. Note that the former has at most 7 nonzero elements per row, while the latter has at most 9 nonzero elements per row.

Figure 6 illustrates the streamlines in and around the cavity door. Figure 6a shows two attached vortices downstream of the spoiler or fence. These vortices separate in Fig. $6 \mathrm{~b}$ and the downstream vortex is shed from the aircraft skin in Figs. $6 \mathrm{c}$ and $6 \mathrm{~d}$.

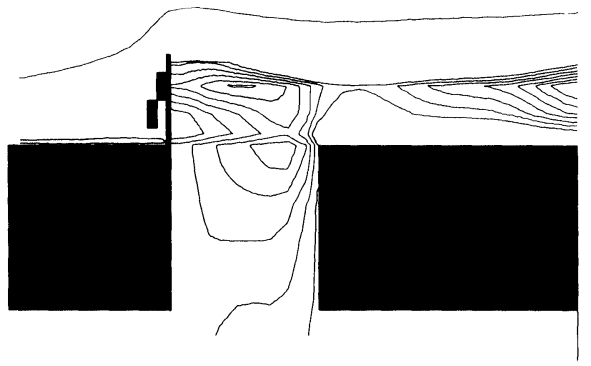

(a)

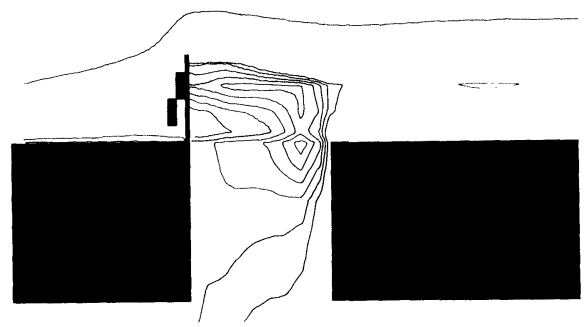

(c)

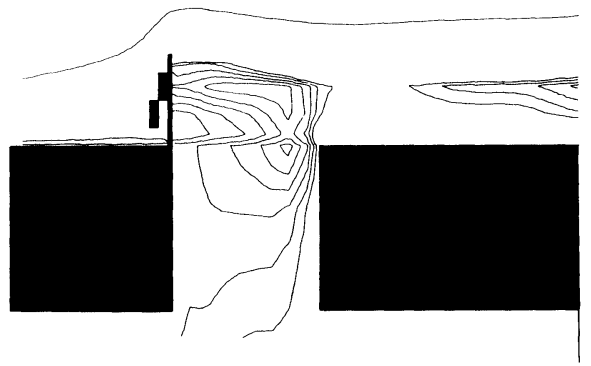

(b)

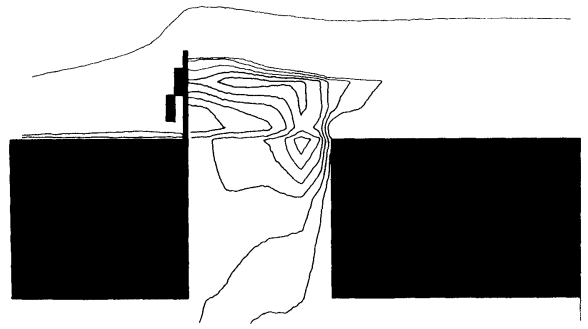

(d)

FIG. 6. (a) Streamlines at times $.005 \mathrm{sec}$; (b) at time $.010 \mathrm{sec}$; (c) at time $.015 \mathrm{sec}$; (d) at time $.020 \mathrm{sec}$. 


\section{REFERENCES}

[1] R. Amit, C. A. Hall ANd T. A. Porsching, An application of network theory to the solution of implicit Navier-Stokes difference equations, J. Comp. Phys., 40 (1981), pp. 183-201.

[2] R. S. Dougall, C. A. HAll AND T. A. PorsChing, DUVAl: A computer program for the numerical solution of two-dimensional, two-phase flow problems, Volumes 1-3, Electric Power Research Institute, Report NP-2099, Palo Alto, CA, 1982.

[3] F. H. HARLOW AND F. W. WELCH, Numerical calculations of time dependent viscous incompressible flow of fluid with a free surface, Phys. Fluids, 8 (1965), p. 2182.

[4] C. A. HALL, Numerical solution of Navier-Stokes problems by the dual variable method, this Journal, 6 (1985), pp. 220-236.

[5] T. A. Porsching, A finite difference method for thermally expandable fluid transients, Nucl. Sci. Eng., 64 (1977), pp. 177-186.

[6] M. Berry, M. Heath, I. Kaneko, M. Lawo, R. J. Plemmons and R. Ward, An algorithm to compute a sparse basis of the null space, Numerische Mathematik, to appear.

[7] N. Deo, G. M. Prabhu AND M. S. Krishnamoorthy, Algorithms for generating fundamental cycles in a graph, ACM Trans. Math. Software, 8 (1982), pp. 26-42. 